\title{
Microsoft Open Government Data Initiative (OGDI), Eye on Earth Case Study
}

\author{
Zdeněk Jiříček and Francesa Di Massimo \\ Microsoft s.r.o., BB Centrum, Building Alpha, Vyskočilova 1461/2a \\ 14000 Praha 4, Czech Republic \\ zdenekj@microsoft.com \\ Microsoft Italy, Centro Direzionale San Felice, Palazzo A \\ Via Rivoltana 13, 20090 Segrate (MI), Italy \\ frandimamicrosoft.com
}

\begin{abstract}
European Open Government Data Initiative is introduced as a free, open-source, cloud-based collection of software assets that government organizations can take advantage of. They can upload and store public data into the Microsoft Cloud, while leveraging the Windows Azure Platform and environment. Important case study of Windows Azure Platform are presented.
\end{abstract}

Keywords: Open Government Data, Cloud Computing, Public Sector Information, Eye on Earth, Windows Azure Platform.

\section{Open Data Driving Open Government}

Open Government is transforming the way organizations across the world handle data and make it available to citizens and communities, and is based on common principles of transparency, participation, and collaboration. Publishing digital data produced and collected by public bodies is a way to realize a piece of Open Government. Overall, this initiative aims at making it easier to publish and reuse a wide variety of public data. This includes mapping, meteorological, legal, traffic, financial, economic, and demographic data. Typically, it doesn't include personal or identifiable information.

\subsection{The Open Government Challenge}

As much of public data sits on different - and commonly incompatible - systems, the main challenge is delivering interoperability to enable its wider distribution and reuse. Interoperability enables governments to unlock data from disparate locations and make it available in an array of e-government services, while taking into consideration security and privacy aspects. By using emerging protocols, smart tagging, semantics, and document format standards, it is now possible to extract data from incompatible systems. New, simple and accessible ways of using the data are already enabling services that benefit both local and global communities, while there's still a lot of potential in front of us. 


\subsection{Why is Public Data So Important?}

In the European Union (EU), the Public sector information (PSI) Directive ${ }^{1}$ sets the legislative framework for the reuse of public information. But Open Government is so much more than a legal provision. Better access to Public Sector Information (PSI) can improve people's quality of life and make their interaction with government much easier. Unlocking the potential of Government data, some analysts are already talking about "Government as a platform". Besides that, using government data can create new businesses and jobs while giving consumers more choice and greater value for money.

\section{The European Open Government Data Initiative (European OGDI) Solution}

European $\mathrm{OGDI}^{2}$ is a free, open-source, cloud-based collection of software assets that government organizations can take advantage of. They can upload and store public data into the Microsoft Cloud ${ }^{3}$, while leveraging the Windows Azure Platform ${ }^{4}$ and environment.

As an open platform, Windows Azure also lets developers use variety of programming languages, such as .NET, PHP, Ruby, Python and more. The European OGDI data repository is continuously being added to and is fully compliant with the PSI Directive principles:

- Availability: Enables governments to publish open data in a reusable format and already includes over 60 different government datasets.

- Transparency: Empowers citizens with more visibility of governments' services.

- Added Value: Enables independent software vendors (ISV) to develop new applications that create more economic value locally.

- Non-discriminatory: Developers working on new applications and services can use the free and customizable source code. It can be used to publish data on the internet in a web-friendly format with easy-to-use, open Application Programming Interfaces (API), which can be accessed from Silverlight, Flash, JavaScript, PHP, Python and Ruby among others.

- Non-exclusivity: Available as open source starter kit on Codeplex portal ${ }^{5}$.

\subsection{Overcoming Barriers towards Open Government}

In Europe, there is untapped economic potential from reusing public data. That's why the European Commission (EC) has issued a PSI directive public consultation to

\footnotetext{
${ }^{1}$ Directive 2003/98/EC of the European Parliament and of the Council of 17 November 2003 on the re-use of public sector information, http://eur-lex.europa.eu/LexUriServ/LexUriServ.do?uri=CELEX:32003L0098:EN:NOT

${ }^{2} \mathrm{http}: / / \mathrm{www}$.govdata.eu/en/index.aspx

${ }^{3} \mathrm{http}: / /$ www.microsoft.com/en-us/cloud/1/home.aspx?fbid=vL91-4EikW6

${ }^{4} \mathrm{http}: / /$ www.microsoft.com/windowsazure/

${ }^{5} \mathrm{http}: / /$ ogdi.codeplex.com
} 
understand more about the barriers. The findings will inform future amendments to the Directive. In summary, Microsoft's view of the current barriers are:

1. Prevalence of open data that is published to application-specific, nonreusable formats that also lack terminology and data consistency.

2. Cost of provisioning and delivery. Data is most often stored on servers within public bodies, not readily available for external, scalable access.

3. Open data frameworks should be publicly available for developers independently on programming languages, together with tools, sample code and documentation to make it easier to re-use the data.

4. More focus is required on publishing open data to the Cloud to improve cost efficiency, to enable reuse features and give non-discriminatory availability for developers and software developers.

\subsection{Why Use the Cloud for Open Data?}

The Cloud $^{6}$ is a highly effective way to publish large amounts of open data quickly and inexpensively without having to invest in a dedicated server infrastructure.

More and more Open Government projects are being hosted in the Cloud. Cloud platforms, such as Windows Azure, provide inherent benefits for Open Government projects, including:

- It supports a variety of standards, languages and protocols, thus realizing specific interoperability ${ }^{7}$ (Interoperability elements ${ }^{8}$ of a cloud platform).

- As a service provider, Microsoft must comply with regulatory requirements of the governmental entities within whose jurisdictions Azure operates, in order to ensure compliance and management of security risks. Windows Azure operates in the Microsoft Global Foundation Services (GFS) infrastructure, portions of which are ISO27001 certified. ISO27001 is recognized worldwide as one of the premiere international information security management standards. Windows Azure is in the process of evaluating further industry certifications. In addition to the internationally recognized ISO27001 standard, Microsoft Corporation is a signatory to Safe Harbor and is committed to fulfill all of its obligations under the Safe Harbor Framework.

- Unlimited amounts of government data can be hosted in the Cloud without additional server hardware and management. Government data becomes even more valuable with greater local relevance. For example, city and local authorities generate rich data about local transportation, construction projects, budgeting and civic facilities.

- The Cloud is highly scalable and you only pay for what is used, so it's ideal for hosting government data which may show varying transaction volumes.

\footnotetext{
${ }^{6} \mathrm{http}: / /$ en.wikipedia.org/wiki/Cloud_computing

${ }^{7}$ http://blogs.msdn.com/b/interoperability/archive/2010/12/27/ quicksteps-to-get-started-with-php-on-windows-azure.aspx

${ }^{8} \mathrm{http}: / /$ blogs.msdn.com/b/interoperability/archive/2010/07/22/ interoperability-elements-of-a-cloud-platform-outlined-at-oscon.aspx
} 
- As government open data is non-PII (Personally Identifiable Information), it is often not constrained by the same data protection issues as, for example, online email or document sharing. As a result, Cloud open data solutions can be hosted internationally.

- $\quad$ Azure Data Market enables government agencies to charge ISV's for the data reuse and consumption and enables ISV's to charge their application users for the services they offer on top of the data. (example: data.gov ${ }^{9}$ ).

\subsection{Open Data Projects Using Microsoft Technologies}

Examples of Open Government initiatives that are already reusing public data, and leveraging Windows Azure and other Microsoft technologies:

- $\quad$ Live map of the London Underground train network in close to real time ${ }^{10}$;

- $\quad$ France health establishments and their locations ${ }^{11}$;

- $\quad$ France Personal services establishments and their locations ${ }^{12}$;

- $\quad$ Parking for Disabled in the City of Rennes ${ }^{13}$;

- Waste collection points in the City of Rennes ${ }^{14}$;

- Trees species in Paris for people allergic to pollen ${ }^{15}$;

- $\quad$ Glass recycling collection points in the City of Paris ${ }^{16}$;

- $\quad$ Easy access to data compiled and Produced by Government Authorities for Swedish Parliament composition ${ }^{17}$;

- $\quad$ Eye on Earth ${ }^{18}$ utilizes Windows Azure, Silverlight, Bing Maps.

- $\quad$ City of Edmonton ${ }^{19}$;

- VanGuide $^{20}$ utilizes Windows Azure, OGDI, Bing Maps, Silverlight, Windows Phone 7.

- $\quad$ Miami $311^{21}$ utilizes Windows Azure, Silverlight, Bing Maps, CRM.

\subsection{Open Data Terms of Use}

The Reuse of the Data published ${ }^{22}$ on OGDI web is regulated by the Terms of Use ${ }^{23}$ that the Government Agency publishing the Data will attach to the Datasets.

\footnotetext{
${ }^{9}$ https://datamarket.azure.com/browse/filter/publisher 55daae4c-da44-438c-aec3690a0d3ba623/

${ }^{10} \mathrm{http} / / /$ traintimes.org.uk/map/tube/

${ }^{11} \mathrm{http}: / /$ www.govdata.eu/samples/INSEE/INSEESante.html

${ }^{12} \mathrm{http} / / / \mathrm{www}$.govdata.eu/samples/INSEE/INSEEServices.html

${ }^{13} \mathrm{http}: / /$ www.govdata.eu/samples/rennes/rennessh.html

${ }^{14} \mathrm{http} / / / \mathrm{www}$. govdata.eu/samples/rennes/rennespaven.html

${ }^{15} \mathrm{http} / / /$ www.govdata.eu/samples/paris/parisarbreseu.html

${ }^{16} \mathrm{http} / / / \mathrm{www}$. govdata.eu/samples/paris/pariscolonnesverre.html

${ }^{17} \mathrm{http}: / / \mathrm{www} .0 \mathrm{ffentligadata.se/Apps.aspx}$

${ }^{18} \mathrm{http} / / / \mathrm{www}$. eyeonearth.eu

${ }^{19} \mathrm{http} / / /$ data.edmonton.ca

${ }^{20} \mathrm{http} / / /$ vanguide.cloudapp.net/

${ }^{21} \mathrm{http}: / /$ miami311.cloudapp.net

${ }^{22} \mathrm{http} / / / \mathrm{dev}$.govdata.eu/
} 


\section{Case Studies}

\subsection{Environmental Data Sharing: Eye on Earth a Cloud-Based, Web 2.0 Solution}

An agency of the European Union, the European Environment Agency (EEA) provides independent and reliable information on the environment for policy makers and the general public. The EEA is working toward raising environmental awareness across Europe by delivering easy-to-understand information about a number of environmental topics-among them, water and air quality. It also encourages citizens to contribute their own observations about the environment around them. Working with Microsoft, it developed the Eye On Earth platform, based on the Windows Azure cloud services operating system. Users can view water or air quality from the 32 member countries of the EEA, using high-definition Bing ${ }^{\circledR}$ Maps.

The EEA has also launched the Environmental Atlas of Europe, which features stories told by eyewitnesses about their first-hand experiences of climate change. Both solutions can help broaden awareness of the impacts of environmental change and help people in Europe make better-informed choices about their environment.

Eye On Earth is supported by cloud services operating system Windows Azure, which works seamlessly with the existing infrastructure, and helps developers quickly deploy new features. The service-based architecture and cloud operating system provide the same level of reliability as an enterprise datacenter, but they offer greater agility, ensuring Eye On Earth can very quickly scale to meet rapid growth in data and traffic.

For WaterWatch, the EEA experiences peak demand during the summer months as people plan their holidays and seek information about the water quality at their destinations. Hundreds of thousands of citizens access the application during its busiest periods, and demand is growing rapidly. AirWatch users are also likely to be increasingly interested in ozone levels in summer when air circulation is more stagnant due to the warmer weather, trapping toxins and pollutants. Cloud technology allows the EEA to easily respond to large-scale peaks in demand.

Bing Maps for Enterprise provides high-resolution satellite images and aerial photography across Europe and beyond. And it's easily customized, so the agency can incorporate its environmental data into the mapping technology with ease.

Data is fed into Microsoft ${ }^{\circledR}$ SQL Azure ${ }^{\mathrm{TM}}$ (a cloud-based database service built on Microsoft SQL Server data management software) every hour. The powerful database supports rapid retrieval of information, making it possible for Eye On Earth to process and deliver data in real time. In addition, the Microsoft Silverlight 3 browser plug-in delivers a seamless media experience, providing users with highly interactive features and Deep Zoom functionality.

Determined to make the application as interoperable as possible, the EEA offers a Microsoft ${ }^{\circledR}$ ASP.NET version of the application allowing everyone to participate, independent of their device. This makes the tools accessible to people with Windows computers using Internet Explorer ${ }^{\circledR}$ and the Firefox browser, Macintosh users with the Safari browser, and people with Linux machines.

With Eye On Earth, the EEA brings the most advanced environmental modeling to citizens across Europe, and encourages them to participate. The data is made available to other interested parties such as urban traffic control, tourism, or healthcare

\footnotetext{
${ }^{23}$ http://dev.govdata.eu/Terms
} 


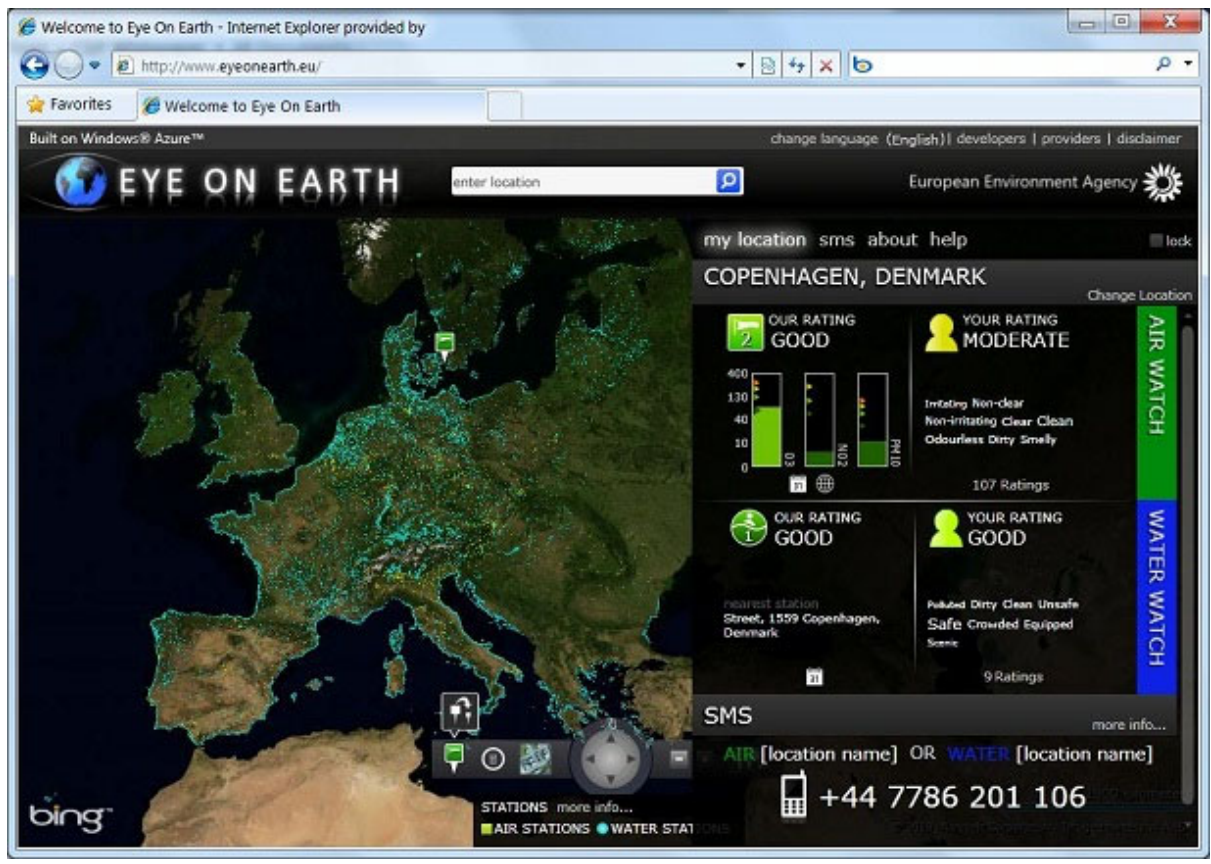

Fig. 1. Eye On Earth platform. Example of Eye On Earth reading, filtered by location for Copenhagen, Denmark.

systems - helping organisations and citizens work together to address climate change, using data that has never before been available on such a scale.

\subsection{Open Data Applications: Rennes - Waste Collection Points}

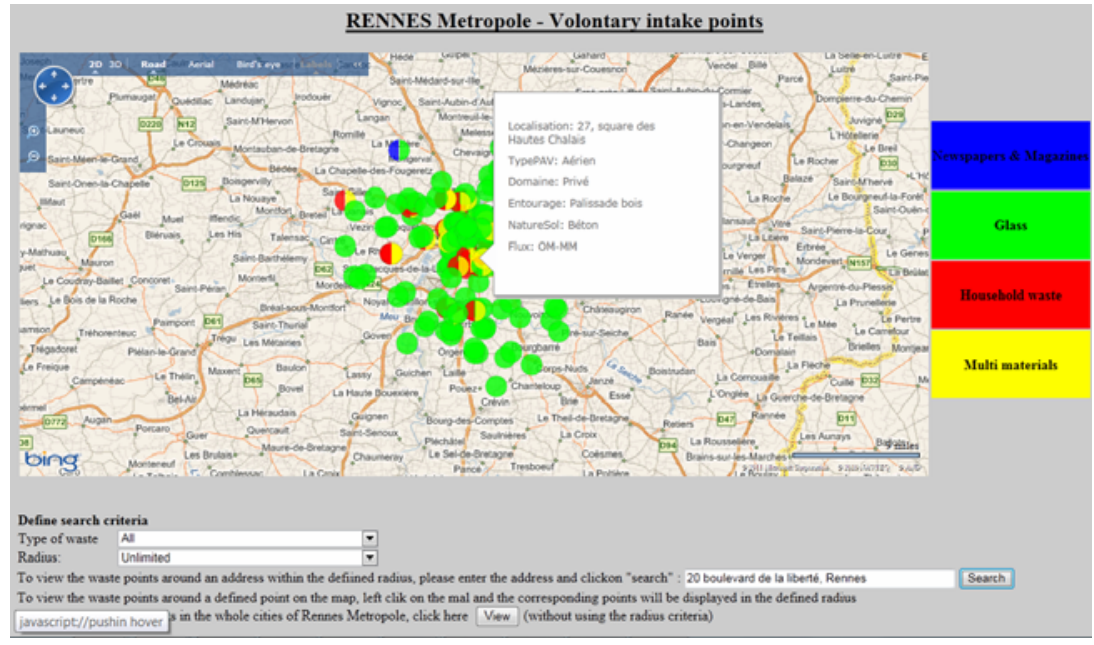

Fig. 2. Rennes - waste collection points 


\subsection{Open Data Applications: Paris - Sorting on Streetside Trees}

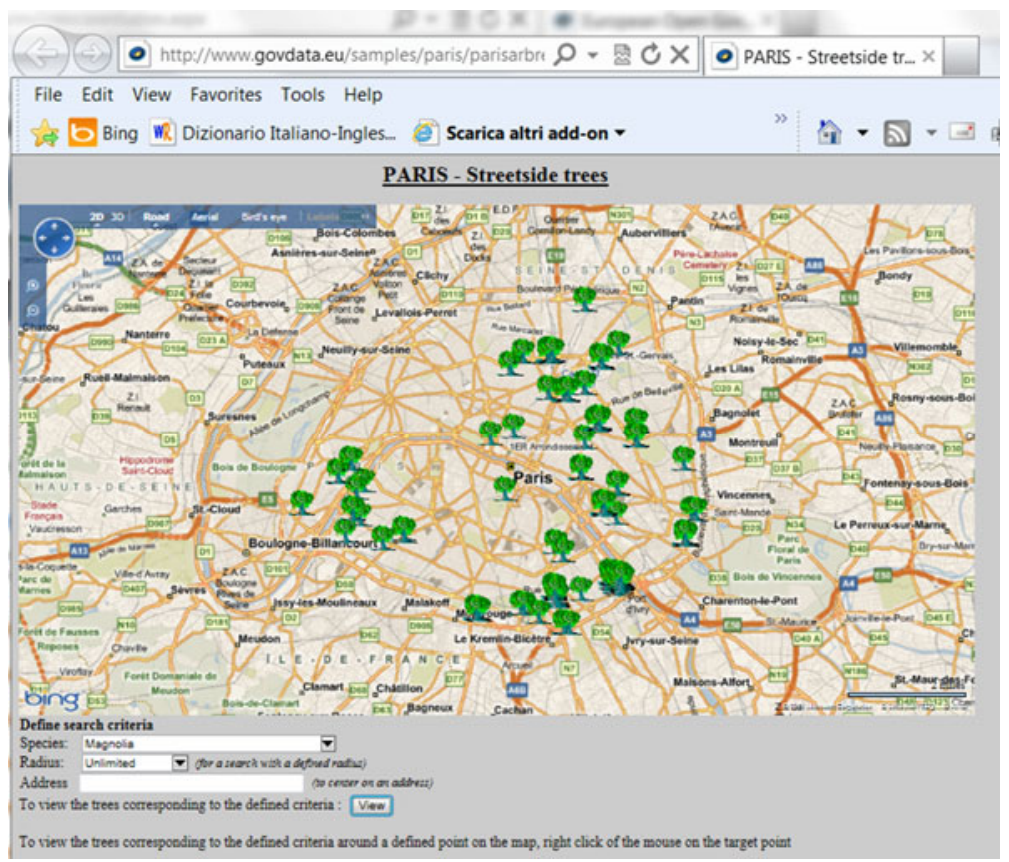

Fig. 3. Paris - sorting on streetside trees

\subsection{Open Data Applications: Paris - Public Toilettes on Windows Phone 7}
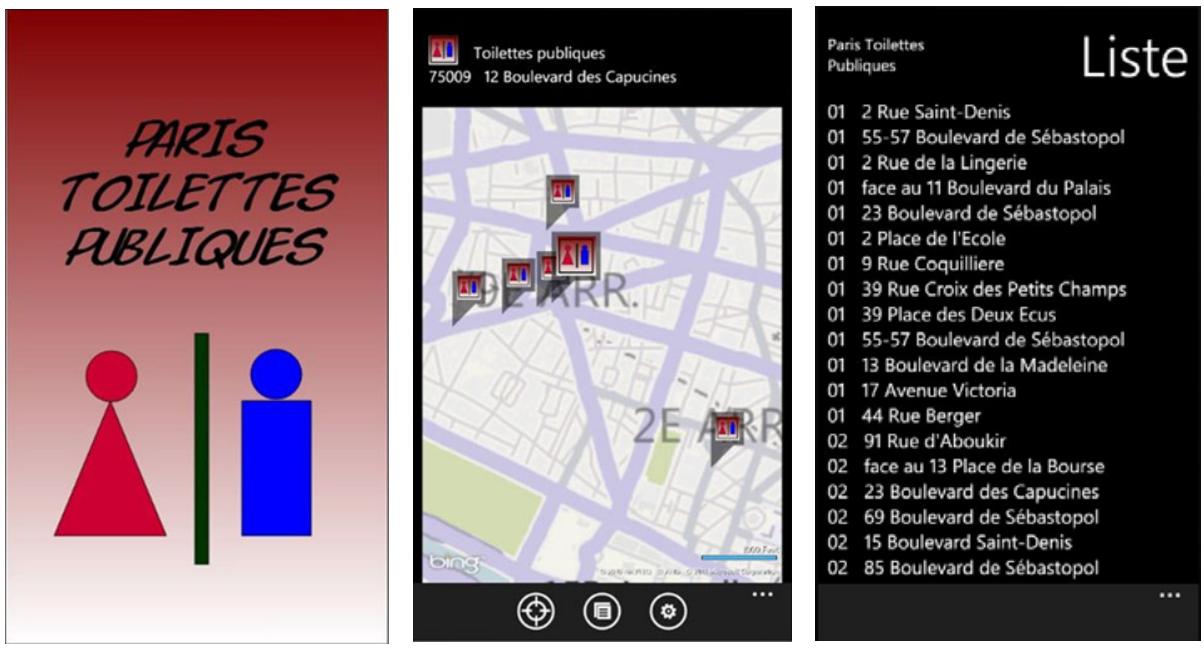

Fig. 4. Paris - public toilettes on Windows Phone 7 